\title{
TransWiki: Supporting Translation Teaching
}

\author{
Robert P. Biuk-Aghai ${ }^{1}$ and Hari Venkatesan ${ }^{2}$ \\ 1 Department of Computer and Information Science \\ Faculty of Science and Technology, University of Macau \\ robertb@umac.mo \\ 2 Department of English \\ Faculty of Social Sciences and Humanities, University of Macau \\ hariv@umac.mo
}

\begin{abstract}
Web-based learning systems have become common in recent years and wikis, websites whose pages anyone can edit, have enabled online collaborative text production. When applied to education, wikis have the potential to facilitate collaborative learning. We have developed a customized wiki system which we have used at our university in teaching translation in collaborative student groups. We report on the design and implementation of our wiki system and an evaluation of its use.
\end{abstract}

Keywords: wiki, translation, collaborative learning.

\section{Introduction}

Computer Supported Collaborative Learning (CSCL) became a reality with advances in technology and notably the advent of the Internet. Computer Aided Instruction, the precursor to CSCL, brought about automation of data dissemination, test-taking, language drills etc. that radically changed the classroom. As technology improved around the mid-1990s, computers and the emerging internet began to be seen as potential tools for creating new learning environments [1, moving away from the mostly uni-directional lecture format of teaching. In 1995 Ward Cunningham created the first wiki [2, a web site whose pages could be read and modified by anyone with access to the internet. Wikis could act as repositories for storage and dissemination of information and the collaborative production of assignments, projects, essays etc. We describe one implementation of a wiki that aims to provide a collaborative learning environment for translation.

The following section provides an overview of related work on using wikis in education. Section 3 then introduces our wiki design and implementation, and Sect. 4 presents an evaluation of the use of our wiki system at our university. Finally, Sect. 5 makes conclusions.

\section{Related Work}

In the field of education, the past few years have witnessed increased adoption of wikis [3]. Generally they are being used in two principal ways: as knowledge repositories for instructors making lecture material and course information 
available 4]; and for the collaborative production of content by students, such as assignments, projects, essays and other assessment material [56]. Examples of the diverse uses which wikis were put to include: collaborative construction of encyclopedia entries by upper secondary school students [6] and university students [5]; collaborative creation of course content [7] or a shared artefact [8] by university students; project management in a project-oriented computer science course 9]; project-oriented product development in an online graduate course [10; essay writing in a university ESL course [11, and for both short and semester-long assignments in a graduate course [12]; and for developing knowledge management processes [13. Wikis have been found to be effective in supporting learning 141516, although the success of wikis may depend on assessment and grades as a form of reward for user contributions [17.

The wiki concept as developed by Cunningham is an open one, where anyone can access and modify any page. This is for example largely the way that the MediaWiki system (www.mediawiki.org) underlying the Wikipedia site works although there are facilities for protecting pages from editing. For educational purposes some degree of access control is usually necessary, e.g. to protect pages that should only be editable by instructors but not by students. Moreover, in order to allow identification of contributions and to prevent changes by outsiders, anonymous editing may need to be disabled and students may be required to login [18. It has also been observed that a single tool integrating all required functions for communication, project management and authoring is preferable to a set of separate tools [10. Basic wiki technology has thus been extended with several different functions specifically for use in education: protecting/locking pages, creating read-only snapshots of an entire wiki site, and others [719].

\section{$3 \quad$ TransWiki}

Our translation wiki system, named TransWiki, is based on the open source MediaWiki system which is the wiki engine of Wikipedia and many other popular wiki sites. MediaWiki is designed for a very open editorial process in which by default every user has read and edit privileges to every page. This can, however, be restricted by locking pages, which in the case of Wikipedia is done whenever edit wars break out on hotly contested pages dealing with controversial topics.

Whereas this open design is suitable for wikis with egalitarian contributors, it is not very suitable for use in education where there are clearly distinct roles of instructor and student. Therefore we have tailored MediaWiki for the use in education through a collection of extension packages that can be added onto an existing MediaWiki installation. An extension essentially provides a plugin that extends the functionality of the MediaWiki system. We have made our extensions available as an open source package named UMEduWiki1. The system structure of TransWiki is shown in Fig. 1. On the server side, the base MediaWiki system is extended by our UMEduWiki extension package, and each of these has its own database (lower portion of Fig. 1, server and database layers). On

\footnotetext{
${ }^{1}$ http://umeduwiki.sourceforge.net/
} 
the client side a unified TransWiki user interface is presented, which consists of both MediaWiki and UMEduWiki portions, and communicates with each of these server components (upper portion of Fig. 1, client layer).

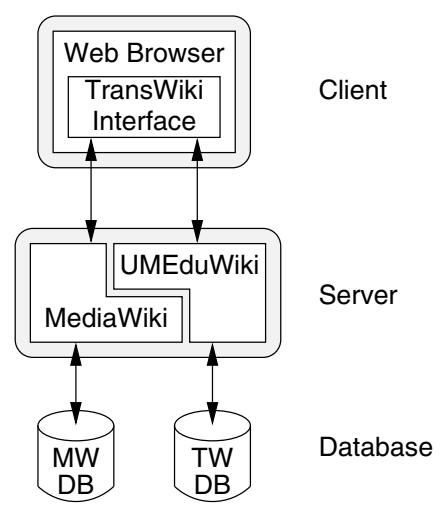

Fig. 1. TransWiki system structure

The base MediaWiki system provides basic wiki functionality: reading and editing of wiki pages, asynchronous online discussion through so-called discussion pages which are attached to wiki article pages, searching, viewing the revision history for any page, as well as several less frequently used wiki editing functions that are normally used by wiki administrators such as locking pages, moving pages, blocking users, and more. TransWiki extends this basic functionality of MediaWiki through following additional functions:

- Group-based access control

- Access log

- Forum-style discussions

- Embedded audio playback

We briefly describe each of these below.

\subsection{Access Control}

Many wikis adopt a "free for all" access model where (almost) every user can perform (almost) every operation. This is not well suited for use in education where instructors and students have distinct roles and need to perform different operations. For example, an instructor may wish to arrange students in separate groups and assign them to do group work. During the process of the group work, the instructor may want to ensure that no group can view the outputs of any other groups' work. However, after the completion of the assigned work the instructor may wish to lock all contributions to prevent further editing (to perform grading of the assignment), and may decide to simultaneously open read 
access to all groups' pages to each other to let them compare their own outcomes with those of their peers. This is the model we have adopted in our translation courses, and it requires a more fine-grained and sophisticated access control than MediaWiki provides.

Our access control component uses a group-based access control model. An instructor or other suitably privileged user can create groups, such as instructor and student groups, or multiple student groups for when a class is divided into several groups. Students are then assigned to these groups. Along with each group we also create a MediaWiki namespace, which allows related pages to be grouped together and be collectively controlled. Students can create pages that belong to their namespace, and instructors can then control access to these pages. Access can be either read or edit. When neither of these is defined then this corresponds to no access rights at all. Access is granted for all the pages of a specified group. For example, an instructor may initially allow groups 1 and 2 only read and edit access to their own pages, then after completing an assignment remove the edit access and at the same time assign read access to each other's pages to share each group's outcome with each other. This kind of access control model may be called medium-grained, lying between the coarse-grained access control of the standard MediaWiki system in which all users are divided into a few roles (user, administrator, sysop etc.) with access permissions having a sitewide scope, and fine-grained access control in other systems where the specific permissions for each user can be set differently on an object-by-object basis. In our experience, this medium-grained access control model combines simplicity with effectiveness and is "fine grained enough" for our purposes. Figure 2 shows the user interface of the privilege management part of our access control panel. The user can select one or more groups on the left (G11, G12, G13, Teacher) to whom to assign the access privilege, select the type of access (read and/or edit), and select to which groups' pages the access should be granted, shown on the right (G11, G12, G13, Teacher).

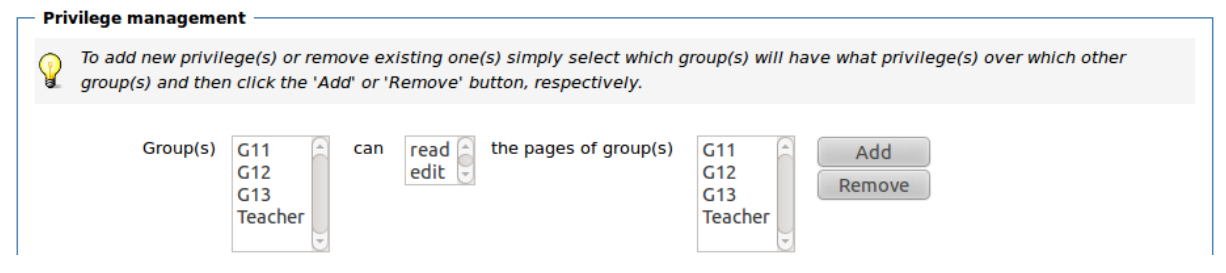

Fig. 2. TransWiki privilege management

\subsection{Access Log}

Wiki engines such as MediaWiki usually record when a user edits a page, including the version, date and time of the edit, a user-provided summary, and some other information. However, MediaWiki does not record when a user views a wiki page. To assess the involvement of students in online activities instructors 
usually wish to know about both reading and editing of wiki pages by students. Therefore we have developed an access log extension as a MediaWiki special page. This special page, which is only visible to instructors, shows who read or edited a page, and when. It provides options to filter the list by user, action, and/or namespace, and limit the number of days to show. It also provides an option to purge old log entries. The Access Log is effective in showing instructors the participation of students in the wiki.

\subsection{Discussion Forum}

Wiki discussion pages in MediaWiki are simply unstructured wiki pages that can be edited by any user, just as regular wiki pages. This is simple but does not always result in a readable record of the discussion as it lacks threading and sorting of discussion statements. Our earliest attempt to remedy this situation was a customized discussion page in which the page was not editable in the usual way but users could only append new posts to the end of the page. We also automatically added a signature line after each post including user name and a timestamp. This was an improvement over the default discussion page as it provided a chronologically sorted record of discussion posts, but all posts were in a single consecutive sequence without any separate threads, making it difficult to keep track of a discussion on a specific topic. Subsequently we developed an entirely new discussion function, with its own separate database tables. This discussion function supports two types of discussion statements: posts and replies. A post effectively starts a new thread, whereas a reply is a statement made in reply to a given post. This limits our discussion forum to just two levels, unlike other discussion forums that allow deeply nested discussions. Our purpose in doing so was to keep the discussions simple while supporting distinct threads. Moreover, we designed the user interface of our discussion forum to closely resemble that of Facebook posts and comments (see Fig. 3). An "agree" link is provided on each post and reply statement, analogous to the "like" link in Facebook. Students are encouraged to click it to indicate agreement with the statement made. This has the effect that one can quickly gauge which proposed translations find widespread acceptance in the group and which not. Clicking on "agree" is also more subtle than directly expressing agreement or disagreement through written statements, which many of our students avoid in order not to offend their fellow students. A lack of "agree's" thus implies disagreement, but without explicitly stating so. As practically all our students are active Facebook users, having a Facebook-like appearance achieves instant familiarity with our discussion function.

In online collaborative translation, discussions play an important role in helping move the collective translation work forward by discussing the suitable way to translate a given text. In teaching translation, instructors need ways to assess not only the outcome of the translation, i.e. the target text, but also the process which led up to this outcome, which is recorded in the online discussion. To facilitate assessment of discussions, we have extended the instructor's view of the discussion forum with two additional functions: a rating function and a tagging 


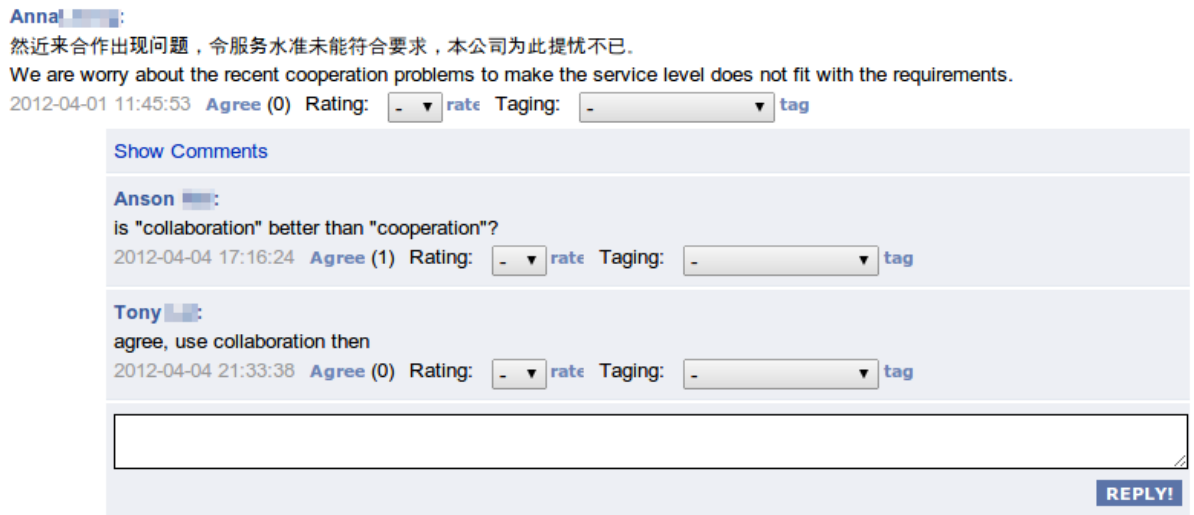

Fig. 3. TransWiki discussion

function. Ratings allow the instructor to indicate how significant a contribution the given discussion post has made and are on a 5-point Likert scale (-2, -1, 0, 1 , 2 ). Tags indicate the type of contribution made, and can be one of the following nine options: Informative, Argumentative, Evaluative, Elicitative, Responsive, Confirmation, Directive, Off task, or Off task technical. Rating and tagging together thus capture both a quantitative as well as a qualitative assessment of each discussion statement.

\subsection{Audio Player}

Our translation teaching includes the teaching of simultaneous interpretation. To integrate the teaching of interpretation into the wiki, we upload mp3 audio files of spoken interpretation exercises to our wiki. A MediaWiki extension that provides a public-domain mp3 player is seamlessly embedded directly in a wiki page to play back these audio files.

\section{Evaluation}

We deployed the TransWiki system at our university in the year 2007 and have used it since in two of our undergraduate translation classes which teach ChineseEnglish translation. Classes were divided into groups of $4-5$ students each. These were composed of students with maximum variety of backgrounds, i.e. local students vs. those from mainland China, students from Chinese medium high schools vs. those from English medium high schools, etc. The underlying assumption in this modus operandi is that collaboration among students in producing the translation will result in interaction, peer review and discussion that would help students better understand the subject. As Tudge observes "...Research based on this model has indicated that social interaction between peers who bring different perspectives to bear upon a problem is a highly effective means 
of inducing cognitive development" 20. Furthermore, the social constructivist perspective on learning that we embrace in the teaching of translation holds that it is "by communicating and negotiating with peers and more experienced (and thus more knowledgeable) others, we acquire a feel for correctness, appropriateness and accuracy, a feel that is grounded in our social experiences..." [21. Thus discussion and collaborative construction of translations are core to the development of translation skills.

Students were given assignments of translating a source text (in either English or Chinese) into the target language (i.e. from English to Chinese, or from Chinese to English). The discussion among group members was to be conducted in the assignment's discussion page inside TransWiki, and at the end when group consensus on the translation had been reached, the resulting target text was to be placed in the assignment wiki page. About 1-2 weeks were allocated for each assignment and about 3-4 assignments were given per semester. After the submission and grading of each assignment, the translated texts from each group were opened for all groups to view and were discussed in class.

At the end of each semester we conducted a survey to assess student attitudes toward the use of TransWiki. These focused on two main areas: (1) collaborative learning and (2) the TransWiki system. Below are the ten survey statements used in the most recent (April 2012) survey.

1. Doing assignments in a group helped improve the quality of translation

2. Discussing assignments exposed me to different ways in which language is used across regions/countries

3. I was able to freely express opinions/disagreement during discussions

4. My group managed to arrive at consensus through discussion

5. I would prefer working on assignments individually

6. Using a web-based platform for discussions was convenient

7. Transwiki was easy to learn and access

8. The discussion page facilitated discussions

9. I would like a live-chat function for discussions

10. I would like a discussion page that reflects changes instantly and automatically

Statements $1-5$ concerned collaborative learning, statements $6-10$ concerned the TransWiki system. A total of 27 students responded whose answers are summarized in Table 1. The results show a strong support for collaborative translation (statements 1 and 2, $81 \%$ and $65 \%$ support, respectively). Even when asked whether students preferred working individually rather than collaboratively (statement 5), only about one third of students expressed this preference, whereas another third expressed the opposite preference and the remaining students were neutral on this issue. Asked about TransWiki, the majority of students supported it in terms of learning and use (statements 6-8, with 58\%, 63\% and $50.0 \%$ support, respectively). Statements 9-10 asked for feedback regarding the discussion function which had attracted student criticism in the past. As before, students favour a more real-time discussion facility, with the larger 
Table 1. TransWiki evaluation results (percentages)

\begin{tabular}{|r|r|r|r|r|r|}
\hline Statement & Strongly Agree & Agree & Neutral & Disagree & Strongly Disagree \\
\hline 1 & 11.1 & 70.4 & 14.8 & 3.7 & 0.0 \\
\hline 2 & 19.2 & 46.2 & 26.9 & 7.7 & 0.0 \\
\hline 3 & 15.4 & 65.4 & 11.5 & 7.7 & 0.0 \\
\hline 4 & 12.0 & 56.0 & 28.0 & 4.0 & 0.0 \\
\hline 5 & 7.7 & 26.9 & 30.8 & 23.1 & 11.5 \\
\hline 6 & 23.1 & 34.6 & 7.7 & 19.2 & 15.4 \\
\hline 7 & 25.9 & 37.0 & 11.1 & 25.9 & 0.0 \\
\hline 8 & 7.7 & 42.3 & 26.9 & 19.2 & 3.8 \\
\hline 9 & 20.0 & 40.0 & 32.0 & 8.0 & 0.0 \\
\hline 10 & 50.0 & 30.8 & 19.2 & 0.0 & 0.0 \\
\hline
\end{tabular}

portion of students expressing a preference for an automatically updating discussion page ( $81 \%$ support). Given this feedback we have in the meantime developed such an instantly updating discussion page using AJAX, similar to the Facebook discussion page, and are deploying it for use in the current semester.

Overall the surveys suggest that working with TransWiki has been generally productive and that the system was well received. Students had complaints regarding the system's speed, especially updating postings on the discussion page. However, use of the discussion page was also appreciated by students who identified lack of peer pressure to agree/conform, as in face-to-face discussions, as one of TransWiki's advantages. The issue of speed was less important where groups allowed discussions to spread out over the entire length of time allowed instead of trying to get together at an appointed time. Such groups generally used the notification function in the wiki which informs students by e-mail once a group member makes a posting. The discussion page is indeed the most important feature of TransWiki as it is at the core of providing a collaborative platform for learning. It also allows all group discussions to be recorded, thus aiding evaluation by the instructor and providing useful insights as to potential problems faced by students. This allows for more focused and individualized teaching.

Regarding disadvantages of the system, a certain portion of students found working with TransWiki cumbersome or difficult. Key areas of complaint included having to log in to the TransWiki page repeatedly, the overall time consumed by the exercise, and discomfort with using computers.

In sum, the use of TransWiki can aid both learning and evaluation. The modus operandi described in this paper also serves as a framework for implementation of constructivist learning environments using TransWiki. The key advantage is the ability to monitor and evaluate the actual collaboration that takes place (if at all). On the other hand ready access to computers and the internet, and being comfortable with using computers over an extended period of time for collaborative assignments are preconditions for successful implementation. 


\section{Conclusions}

We have presented TransWiki, a customized wiki system for use in translation teaching. Our design is based on the existing MediaWiki system, extended with custom code developed by us. In the translation community it is well established that collaborative translation produces better quality results than individual translation. The challenge, however, lies in actually bringing about collaboration among a group of translators. Our experience with our students confirms that TransWiki facilitates online collaboration and discussion on translation. Through TransWiki students feel free to express their opinions and to collaborate, which is particularly relevant for the Asian context where students often hold back their opinions in face-to-face settings but feel more free to express themselves online. TransWiki's features enable instructors to provide separate working areas for separate student groups, enabling focused group work. Moreover, having a digital record not only of the finished translations but also of the communication within each group that led to the translation allows instructors to review and assess both process and outcome of translation, and to identify how learning has occurred. Thus TransWiki is both an enabling platform for collaborative translation and a pedagogical tool for translation teaching.

The design and implementation of TransWiki are not limited to use in translation, however. Other online collaborative work that involves joint construction of content should equally benefit from the features provided by TransWiki.

\section{References}

1. Alavi, M.: Computer-mediated collaborative learning: an empirical evaluation. MIS Quarterly 18(2), 159-174 (1994)

2. Leuf, B., Cunningham, W.: The Wiki Way: Quick Collaboration on the Web. Addison-Wesley Longman Publishing Co., Inc. (2001)

3. Pusey, P., Meiselwitz, G.: Heuristics for Implementation of Wiki Technology in Higher Education Learning. In: Ozok, A.A., Zaphiris, P. (eds.) OCSC 2009. LNCS, vol. 5621, pp. 507-514. Springer, Heidelberg (2009)

4. Schwartz, L., Clark, S., Cossarin, M., Rudolph, J.: Educational wikis: features and selection criteria. The International Review of Research in Open and Distance Learning 5(1) (2004)

5. Bruns, A., Humphreys, S.: Wikis in teaching and assessment: the m/cyclopedia project. In: Proceedings of the 2005 International Symposium on Wikis, pp. 2532. ACM, New York (2005)

6. Lund, A., Smørdal, O.: Is there a space for the teacher in a wiki? In: Proceedings of the 2006 International Symposium on Wikis, pp. 37-46. ACM, New York (2006)

7. Wang, C.M., Turner, D.: Extending the wiki paradigm for use in the classroom. In: Proceedings of the International Conference on Information Technology: Coding and Computing (ITCC 2004), vol. 2, IEEE Computer Society, Washington, DC (2004) 
8. Hampel, T., Selke, H., Vitt, S.: Deployment of simple user-centered collaborative technologies in educational institutions - experiences and requirements. In: Proc. 14th IEEE International Workshops on Enabling Technologies: Infrastructure for Collaborative Enterprise, pp. 207-214. IEEE Computer Society, Washington, DC (2005)

9. Xu, L.: Project the wiki way: using wiki for computer science course project management. J. Comput. Sci. Coll. 22(6), 109-116 (2007)

10. Icaza, J., Heredia, Y., Borch, O.: Project oriented immersion learning: Method and results. In: 6th International Conference on Information Technology Based Higher Education and Training (ITHET 2005), pp. T4A-7. IEEE (2005)

11. Wang, H.C., Lu, C.H., Yang, J.Y., Hu, H.W., Chiou, G.F., Chiang, Y.T.: An empirical exploration of using Wiki in an English as a second language course. In: Proc. Fifth IEEE International Conference on Advanced Learning Technologies, pp. 155-157. IEEE Computer Society, Washington, DC (2005)

12. Bower, M., Woo, K., Roberts, M., Watters, P.: Wiki pedagogy-a tale of two wikis. In: 7th International Conference on Information Technology Based Higher Education and Training (ITHET 2006), pp. 191-202. IEEE (2006)

13. Biasutti, M., El-Deghaidy, H.: Using wiki in teacher education: Impact on knowledge management processes and student satisfaction. Comput. Educ. 59(3), 861$872(2012)$

14. Šerbec, I.N., Strnad, M., Rugelj, J.: Assessment of wiki-supported collaborative learning in higher education. In: Proceedings of the 9th International Conference on Information Technology Based Higher Education and Training, pp. 79-85. IEEE Press, Piscataway (2010)

15. Tsai, W.T., Li, W., Elston, J., Chen, Y.: Collaborative learning using wiki web sites for computer science undergraduate education: A case study. IEEE Trans. on Educ. 54(1), 114-124 (2011)

16. Tselios, N., Altanopoulou, P., Katsanos, C.: Effectiveness of a framed wiki-based learning activity in the context of HCI education. In: Proceedings of the 2011 15th Panhellenic Conference on Informatics, pp. 368-372. IEEE Computer Society, Washington, DC (2011)

17. Ebner, M., Kickmeier-Rust, M., Holzinger, A.: Utilizing wiki-systems in higher education classes: a chance for universal access? Univers. Access Inf. Soc. 7(4), 199-207 (2008)

18. Raitman, R., Ngo, L., Augar, N.: Security in the online e-learning environment. In: Proc. Fifth IEEE International Conference on Advanced Learning Technologies, pp. 702-706. IEEE Computer Society, Washington, DC (2005)

19. Elrufaie, E., Turner, D.A.: A wiki paradigm for use in IT courses. In: Proceedings of the International Conference on Information Technology: Coding and Computing (ITCC 2005), vol. 2, pp. 770-771. IEEE Computer Society, Washington, DC (2005)

20. Tudge, J.: Vygotsky, the zone of proximal development, and peer collaboration: Implications for classroom practice. In: Moll, L.C. (ed.) Vygotsky and Education: Instructional Implications and Applications of Sociohistorical Psychology, pp. 155172. Cambridge University Press (1992)

21. Király, D.C.: A social constructivist approach to translator education: Empowerment from theory to practice. St. Jerome Pub. (2000) 